\title{
NEO follow-up, recovery and precovery campaigns at the ESA NEO Coordination Centre
}

\author{
Marco Micheli ${ }^{1,2,3}$, Detlef Koschny ${ }^{1,4,5}$, Gerhard Drolshagen ${ }^{1,4}$, \\ Ettore Perozzi ${ }^{1,3,6}$ and Barbara Borgia ${ }^{1,2,3}$ \\ ${ }^{1}$ ESA NEO Coordination Centre, Frascati (RM), Italy \\ email: marco.micheli@esa.int \\ ${ }^{2}$ SpaceDyS s.r.l., Cascina (PI), Italy \\ ${ }^{3}$ INAF-IAPS, Roma (RM), Italy \\ ${ }^{4}$ ESA ESTEC, Noordwijk, The Netherlands \\ ${ }^{5}$ Chair of Astronautics, Technical Univ. Munich, Garching, Germany \\ ${ }^{6}$ Deimos Space, Bucharest, Romania
}

\begin{abstract}
The NEO Coordination Centre (NEOCC) has been established within the framework of the ESA Space Situational Awareness (SSA) Programme. Among its tasks are the coordination of observational activities and the distribution of up-to-date information on NEOs through its web portal.

The Centre is directly involved in observational campaigns with various telescopes, including ESO's VLT and ESA's OGS telescope. We are also developing a network of collaborating observatories, with a variety of capabilities, which are alerted when an important observational opportunity arises.

From a service perspective, the system hosted at the NEOCC collects information on NEOs produced by European services and makes it available to users, with a focus on objects with possible collisions with the Earth. Among the tools provided via our portal are the Risk List of all known NEOs with impact solutions, and the Priority List, which allows observers to identify NEOs in most urgent need of observations.
\end{abstract}

Keywords. minor planets, asteroids

\section{Introduction}

The SSA-NEO program is one of the three components of ESA's Space Situational Awareness (SSA) program, a large project focusing on the assessment, monitoring and prevention of risks to ground- and space-based human assets coming from space. Over the past few years, the NEO segment of the SSA program has grown into a complex system of various components, which all work together toward the goal of a complete European system of asteroid observation, data analysis and risk mitigation activities.

Among the goals of the program are:

- The provision of NEO information and the coordination of observations via the NEO Coordination Centre (NEOCC)

- The development of a European wide-field survey

- The development of a system to issue warnings and alerts to civilian authorities in case of a credible impact threat

- The support of NEO mitigation measures

- International coordination on NEO-related topics (e.g. SMPAG, IAWN) 
In the following we will focus on the activities of the NEO Coordination Centre, explaining its role, activities and services, and its contributions to the global NEO community.

\subsection{The NEO Coordination Centre}

The NEO Coordination Centre, located in the ESA ESRIN establishment in Frascati, near Rome, functions as the European coordination node for NEO-related activities, both observational and data-oriented. Founded in 2013, it has progressively developed activities and capabilities in two general areas:

- Coordination of observational campaigns and data collection

- Computation, collection and distribution of information about NEOs and impact risk

To address the first goal, the Centre both directly coordinates and executes observations of NEOs, with a particular focus on astrometry of objects with non-zero impact probability with Earth (objects with the so called Virtual Impactor solutions, or VIs). It also plans to serve as an online repository for all data and image products obtained through our cooperating assets.

On the informational side, the Centre relies on well-known European data analysis centers, such as NEODyS and EARN, to obtain and distribute information on the NEO risk, both to the general public and to the scientific community. It also directly interacts with outside users by responding to direct queries and information requests, both from the media and the public at large.

\section{Observational activities}

One of the primary goals of the coordination activities of the NEOCC is to ensure that the most important objects, such as those with non-zero impact probability, receive adequate observational coverage any time they are observable. This goal is pursued by two parallel ways, via direct observations managed by the NEOCC staff and through the establishment of international collaborations with other astronomers.

Some of the results and methods of our observational activities have been discussed in Micheli et al. (2014). The following sections will summarize the main assets to which we have access, and our approach to observational coordination.

\section{1. $V L T$}

The most powerful facility to which we have routine direct access is ESO's Very Large Telescope (VLT), equipped with the FORS2 imager. Thanks to an agreement between ESA and ESO, in collaboration with Olivier Hainaut we currently manage 11 hours of telescope time each semester, dedicated to astrometric follow-up of objects with impact solutions. Thanks to its $8.2 \mathrm{~m}$ aperture, VLT allows us to detect objects as faint as $V \sim 27$, and extract astrometric positions with $\sim 0.1^{\prime \prime}$ accuracy.

We have used our VLT time to successfully obtain follow-up and recovery observations of more than 30 objects with VIs between 2014 and 2015, most of which were removed from the Risk List thanks to our observations. An example of one such detection is presented in Fig. 1.

The only limitation of this instrumental setting is the moderately small $7^{\prime}$ field of view $(\mathrm{FoV})$ of FORS2; VLT is therefore not ideal to perform wide-field recoveries of large-uncertainty NEOs, that are sometimes easier to find with smaller apertures, if compensated by a larger angular coverage. 


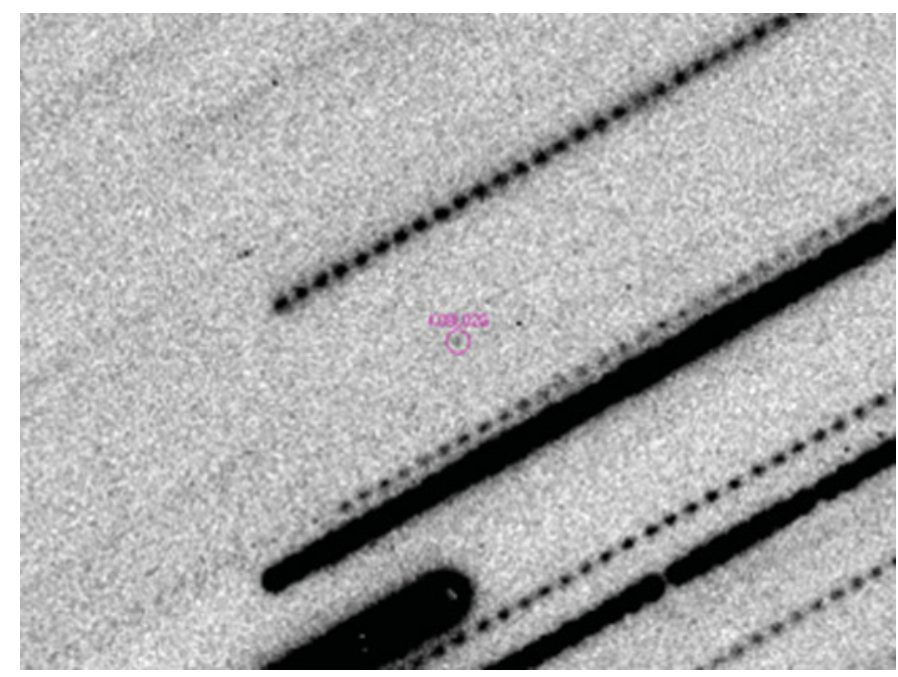

Figure 1. Detection of 2008 LG2 with VLT and the FORS2 camera (unfiltered), obtained on 2015 July 8 with $\sim 60$ min of integration time.

\subsection{ESA OGS}

ESA's Optical Ground Station (OGS) with its wide-field Space Debris Camera, located on Tenerife, Canary Islands, is an ideal resource for wide-field observations. Although the telescope is much smaller than VLT, with a primary mirror of only $1 \mathrm{~m}$, its camera has a FoV of $\sim 47^{\prime}$, which easily covers linear uncertainties of a few degrees with just a few fields, down to $V \sim 22$.

The telescope is usually available to our team 4 to 6 nights per month, around new Moon. We routinely use it to perform immediate follow-up of NEO Confirmation Page (NEOCP) objects, and targeted recoveries of NEOs that are becoming observable at their second apparition, especially those with large positional uncertainties. Part of the time is also dedicated to a small-scale survey, called TOTAS and managed by Matthias Busch (Koschny \& Busch (2014)), which has been successful in discovering about a dozen new NEOs (including one with impact solutions) and two comets over the past few years.

\subsection{Other cooperating telescopes}

Over the past couple of years, ESA has begun to sponsor some small-scale observational projects in Europe, guaranteeing support to smaller telescopes dedicated to NEO followup via service level agreements. Among these facilities are the $1.06 \mathrm{~m}$ reflector of the Klet Observatory in the Czech Republic, the $1.2 \mathrm{~m}$ reflector in Calar Alto, Spain, and more recently the $0.8 \mathrm{~m}$ Schmidt located at the same site.

The latter telescope in particular has just been refurbished and made available for asteroid work, with a remote-control interface. The first test observations are being performed at the time of this writing, and the site has been recently assigned IAU code Z84.

\subsection{Global collaborations}

Although the telescopic resources presented above are often sufficient to perform our own follow-up and recovery observations of targets deemed important by the Centre, we sometimes notice objects that require specific instruments or expertise to be properly observed. To address these cases and make sure that good observational opportunities are not missed, we've developed an extended network of contacts all over the world, 


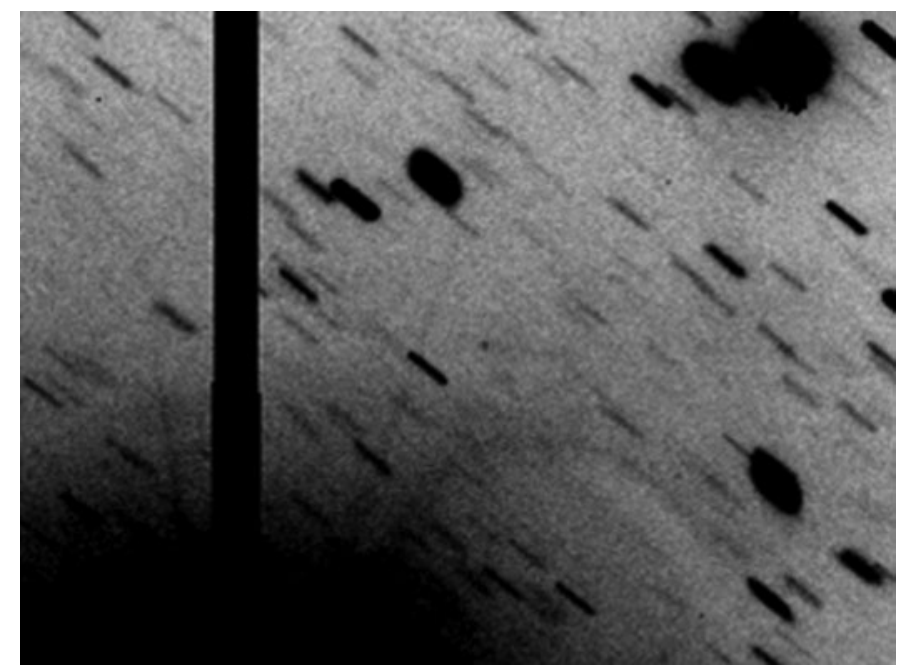

Figure 2. Detection of 2014 KC46 with LBT through its blue-sensitive CCD, obtained on 2014 October 30 with $\sim 30$ min of integration time. The positional uncertainty at the time of the observations spanned all three chips of the camera.

including professional and amateur astronomers located in various geographical locations, and with access to a variety of observational technologies. These collaborators are often alerted when an observational opportunity particularly suited for their expertise arises. In these cases, the Centre is available to offer them all the necessary support to guarantee that the scientific outcome of the observation is maximized. We are always interested in expanding our collaborations and establishing new fruitful scientific connections, and we invite anybody who is interested to contact us directly.

A particularly successful collaboration has been established with the Italian partnership of the Large Binocular Telescope (LBT), through the Italian Institute for Astrophysics (INAF). LBT is an exceptional resource for asteroid observations, due to its peculiar capability to simultaneously observe the same field with two comounted $8.4 \mathrm{~m}$ telescopes. Its Large Binocular Cameras (LBCs) have a large FoV $\left(\sim 27^{\prime}\right)$ and extremely good sensitivity, which give LBT a great potential to perform recoveries of faint NEOs with even moderately large ephemeris uncertainties. Fig. 2 shows the result of our first test with LBT, the recovery of possible impactor $2014 \mathrm{KC} 46$ at $V=26$.

In addition to direct collaborations with individuals and research centers, the Centre is also in contact with existing networks of telescopes and observers, such as EURONEAR, the Gaia FUN-SSO network, and the LCOGT network.

\subsection{Precoveries}

One topic to which the NEOCC has been paying particular attention is the search for precovery observations of NEOs in public image repositories. The term precovery is used to indicate a serendipitous observation of an object before its actual discovery, taken at a time when the object was still unknown and usually difficult to identify in the image. Precoveries have an extremely interesting potential in the field of NEO observations, because they can easily provide significant extension of the observed arc for an object, without any investment of telescope time. Furthermore, in some cases they are the only available option to obtain additional observations of lost objects that cannot be located again because their positional uncertainties are already prohibitive. 
To contribute to the NEO precovery effort, the NEOCC is currently developing an online archive in order to make all our images available to the public. This archive will be indexed by the CADC Search System (Gwyn et al. (2012)), and will be available through it to the whole community.

\section{Data distribution}

One of the main goals of the NEOCC is the development and maintenance of a web portal providing up-to-date and reliable information on NEOs to the community. Our services are accessible at http://neo.ssa.esa.int, and are frequently updated to reflect new discoveries and information from the literature. Some of the services are comparable to those offered by NASA via their web portals at JPL (neo.jpl.nasa.gov and ssd.jpl.nasa.gov), while some others have been evolved in different ways and provide complementary information to the user.

Some of the products are generated by our own system, while others are obtained through agreements with other European entities, such as NEODyS or EARN.

\subsection{The Risk List}

The most important page available on the portal is the so called Risk List, the tabulation of all known asteroids with non-zero impact probability. Our list mirrors the same information computed by the NEODyS group in Pisa, Italy, but provides the data in a way that is easier to understand for the general public and the non-scientific user. For each asteroid in the list, the date and the impact probability of the most likely possible impact is presented, together with accessory data such as the Palermo Scale (Chesley et al. (2002)), the encounter velocity and an estimate of the diameter of the object. When an object has a published diameter in the literature its value is used, while if no direct measurement is available the size is indirectly estimated from the absolute magnitude, with assumptions on the albedo.

As of the date of this writing, the Risk list contains more than 500 objects, out of a population of about 13000 known NEOs. Most of them have impacts computed for a timespan of 100 years in the future, apart for a few exceptional cases where the impact monitoring has been extended to longer periods. The highest-rated object in terms of Palermo Scale is currently (410777) 2009 FD, with a value of -0.43 and an impact probability of $\sim 1 / 369$ for an encounter on 2185 March 29.8 UT (see Spoto et al. (2014) for details on how this particular object was analyzed). However, the highest impact probability is for 2010 RF12, a ten-meter object with a probability of $\sim 1 / 11$ to impact the Earth in 2095, although with negligible consequences given its very small size.

\subsection{The Priority List}

The second important service provided by the system is the Priority List, a ranked list of the observable NEOs in most urgent need of astrometric follow-up. The list, based on the algorithms from Boattini et al. (2007), prioritizes observable objects in four different classes, from urgent to low-priority, on the basis of how important a current observation would be to improve the future observability of the object, and prevent it from becoming lost. The service was originally provided by the Spaceguard Foundation, and it is now entirely integrated into the NEOCC system.

The main list covers objects with current magnitude $V<22$ and elongation $>40^{\circ}$, addressing the needs of observers with meter-class telescopes (or smaller). There is however a faint version of the list, based on the same algorithms, which extends down to $V=25$ and smaller elongations. 


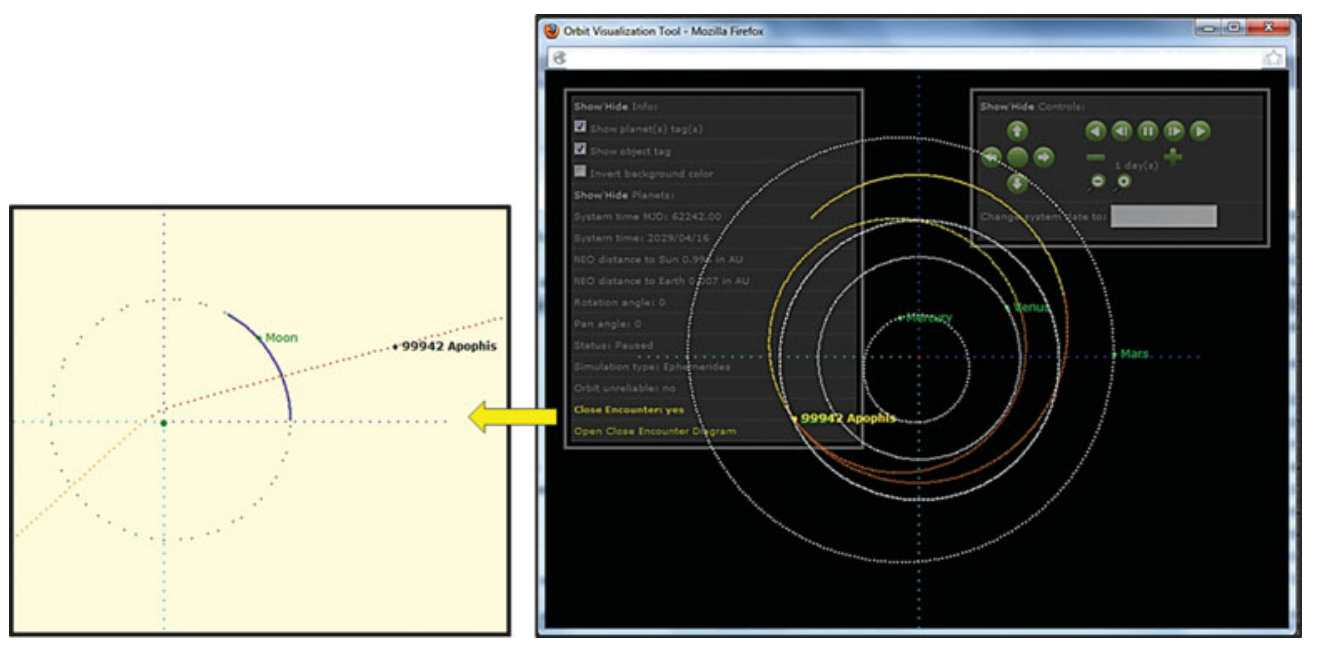

Figure 3. The NEOCC orbit visualization tool showing the perturbed trajectory of asteroid (99942) Apophis before and after its 2029 encounter with the Earth in a heliocentric (right) and geocentric (left) reference frame.

\subsection{The List of Close Approaches}

This page presents a list of past and upcoming close approaches of known NEOs within 20 lunar distances. For each object, the time and distance of fly-by are given, together with the object diameter. An upcoming update to the list will include the peak magnitude reached by the object during the apparition, a useful parameter in the preparation of observational proposals for professional telescopes.

\subsection{News and Newsletter}

In addition to the services presented above, which are mostly data-oriented, the NEOCC also strives to provide correct and updated information to the general public and nonspecialists. To this end, the Centre regularly publishes news articles on important events of NEO science, to inform the public on the developments of the field. Occasionally a news item may cover a particularly important result or development of the Centre.

The program also issues a monthly two-page newsletter which provides concise information on the most important NEO events of the month, including past and future close approaches, events or scientific conferences in the field, and interesting bits of information and images. The monthly newsletter is accessible through our portal or can be received by e-mail with a free subscription.

\subsection{Orbital and Physical Information Database}

Another important resource available through the NEOCC portal is a fully searchable database with orbital information of all known small solar system bodies, plus physical information on NEOs ingested from the EARN project. The resulting tool allows the user to combine dynamical and compositional queries, offering features rarely found in other comparable online tools.

In addition to the search capabilities, the system provides an Orbit Visualization Tool that displays the orbit of the object in relation to the rest of the Solar System. The plotted orbit is fully perturbed and evolves with time; during a close approach with Earth it is also possible to zoom on the Earth-Moon system and follow the details of the encounter in a geocentric frame (Fig. 3). 
Table 1. List of objects removed from the Risk List in the last 2 years thanks to observations obtained by the NEOCC.

\begin{tabular}{|c|c|c|c|c|}
\hline Object & Date & $\mathrm{PS}_{0}$ & | Telescope & | Instrument \\
\hline 2007 UW 1 & 2013-11-28 & -3.4 & $\mathrm{CFHT}$ & MegaCam \\
\hline 2013 XE2 & $2013-12-10$ & -4.0 & PS1 & GPC \\
\hline 2008 CK 70 & 2013-12-18 & -3.1 & $\mathrm{CFHT}$ & MegaCam \\
\hline 2013 ВР73 & $2013-12-20$ & -3.8 & SDSS & \\
\hline 2013 YC & 2014-01-22 & -2.9 & VLT (UT1) & FORS2 \\
\hline 2014 BD33 & 2014-01-29 & -4.2 & PS1 & GPC \\
\hline 2004 BX159 & 2014-02-18 & -4.5 & $\mathrm{CFHT}$ & MegaCam \\
\hline 2014 AF16 & 2014-03-11 & -2.4 & VLT (UT1) & FORS2 \\
\hline 2012 HP13 & 2014-04-09 & -6.6 & VLT (UT1) & FORS2 \\
\hline 2014 DN112 & 2014-05-01 & -3.6 & VLT (UT1) & FORS2 \\
\hline 2014 HM129 & 2014-05-22 & -4.2 & VLT (UT1) & FORS2 \\
\hline 2014 HM 187 & 2014-05-28 & -4.5 & VLT (UT1) & FORS2 \\
\hline 2012 VU76 & 2014-06-09 & -6.1 & VLT (UT1) & FORS2 \\
\hline 2013 YD 48 & 2014-06-30 & -4.8 & VLT (UT1) & FORS2 \\
\hline 2014 LU27 & 2014-07-17 & -2.4 & PS1 & GPC \\
\hline 2014 PB58 & 2014-08-12 & -4.5 & PS1 & GPC \\
\hline 2014 QF 392 & 2014-08-14 & -8.0 & PS1 & GPC \\
\hline 2014 QJ392 & 2014-08-14 & -6.1 & PS1 & GPC \\
\hline 2014 RC & 2014-09-04 & -7.0 & PS1 & GPC \\
\hline 2014 KC46 & 2014-10-30 & -4.1 & LBT & LBC \\
\hline 2014 W V 363 & 2014-12-01 & -3.4 & PS1 & GPC \\
\hline 2014 XL7 & 2015-01-15 & -3.0 & VLT (UT1) & FORS2 \\
\hline 2003 LN6 & $2015-01-23$ & -5.2 & VLT (UT1) & FORS2 \\
\hline 2015 BU92 & 2015-01-27 & -2.9 & $\mathrm{LCOGT}$ OGG (FTN) & Spectral \\
\hline 2014 XM7 & 2015-02-09 & -6.5 & PS1 & GPC \\
\hline 2015 DA54 & 2015-02-26 & -5.4 & PS1 & GPC \\
\hline 2015 DF 198 & 2015-02-26 & -5.4 & PS1 & GPC \\
\hline 2014 NG65 & $2015-03-25$ & -4.5 & PS1 & GPC \\
\hline 2014 WP362 & 2015-04-10 & -4.8 & VLT (UT1) & FORS2 \\
\hline 2008 LG2 & 2015-06-16 & -5.8 & VLT (UT1) & FORS2 \\
\hline 2015 KL157 & $2015-07-13$ & -3.9 & OGS & SDC \\
\hline 2015 OL35 & 2015-08-02 & -3.9 & PS1 & GPC \\
\hline 2015 PR228 & $2015-08-18$ & -3.6 & PS1 & GPC \\
\hline 2015 PK57 & 2015-08-18 & -9.7 & PS1 & GPC \\
\hline 2000 UK 11 & $2015-08-26$ & -5.6 & VLT (UT1) & FORS2 \\
\hline 2015 RA36 & 2015-09-14 & -4.5 & OASI & - \\
\hline
\end{tabular}

Notes:

$\mathrm{PS}_{0}$ indicates the Palermo Scale of the object before the observations were included in the orbital solution.

\subsection{Other services}

Among the other services offered through the web portal, it is worth mentioning a statistics page on NEO discoveries, outreach material and an interesting and complete Chronology of NEO science, kindly provided and maintained by Karel van der Hucht.

A few additional services will become available soon, including a database of astronomical images obtained by telescopes in our system (see below for more details), a searchable database of bright fireballs, and tools developed in other parallel contracts.

\section{Conclusions}

The NEOCC system, established two years ago, has already grown into a complex structure and produced valuable results.

From an observational perspective, the Centre has been actively producing observations of important targets, especially possible impactors. Most of these observations have been critical in clarifying the impact probabilities of many NEOs, and have resulted in the removal of dozens of NEOs from the Risk List (see Table 1 for a summary of these observations). A significant fraction of these results have been achieved thanks to the extensive collaboration network established by the Centre, or through a dedicated precovery search effort in image databases. 
As a data provider, the portal already includes a variety of information, and it is constantly growing with the addition of new services and resources. Many additional features are upcoming and other services are planned for the next few years.

In the future, the NEOCC plans to continue and increase its activities within the framework of the ESA SSA program, which is currently expanding to include more resources (such as a wide field survey), to develop a system to issue warnings and alerts to civil authorities in case of a credible impact threat, and to support NEO mitigation measures in an international setting (e.g. through the UN-mandated International Asteroid Warning Network and Space Missions Planning Advisory Group).

\section{References}

Boattini, A., D'Abramo, G., Valsecchi, G. B., \& Carusi, A. 2007, Earth Moon and Planets, 100, 31

Chesley, S. R., Chodas, P. W., Milani, A., Valsecchi, G. B., \& Yeomans, D. K. 2002, Icarus, 159, 423

Gwyn, S. D. J., Hill, N., \& Kavelaars, J. J. 2007, Earth Moon and Planets, 124, 579

Koschny, D. \& Busch, M. 2014, Asteroids, Comets, Meteors 2014, 283

Micheli, M., Koschny, D., Drolshagen, G., Hainaut, O., \& Bernardi, F. 2014, Earth Moon and Planets, 113, 1

Spoto, F., Milani, A., Farnocchia, D., Chesley, S. R., Micheli, M., Valsecchi, G. B., Perna, D., \& Hainaut, O. 2014, $A \mathscr{G} A, 574,100$

\section{Discussion}

MorBIDELLI: A new NEO population model has been developed under ESA contract, and will be avaiable soon. Can you confirm that it will be distributed via the NEOCC website?

Micheli: Yes, the new population model will be one of the new services available in the upcoming version of our web portal. 